DESY 15-033

\title{
On the efficient numerical solution of lattice systems with low-order couplings
}

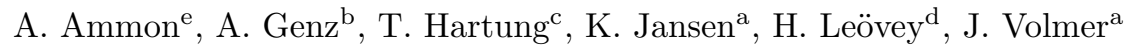 \\ ${ }^{a}$ NIC, DESY Zeuthen, Platanenallee 6, D-15738 Zeuthen, Germany \\ ${ }^{b}$ Department of Mathematics, Washington State University, Pullman, WA 99164-3113 USA \\ ${ }^{c}$ Department of Mathematics, King's College London, Strand, London WC2R 2LS, United \\ Kingdom \\ ${ }^{d}$ Institut für Mathematik, Humboldt-Universität zu Berlin, Unter den Linden 6, D-10099 \\ Berlin \\ ${ }^{e} \mathrm{OAKLABS}$ GmbH, Neuendorfstr. 20b, 16761 Hennigsdorf, Germany
}

\begin{abstract}
We apply the Quasi Monte Carlo (QMC) and recursive numerical integration methods to evaluate the Euclidean, discretized time path-integral for the quantum mechanical anharmonic oscillator and a topological quantum mechanical rotor model. For the anharmonic oscillator both methods outperform standard Markov Chain Monte Carlo methods and show a significantly improved error scaling. For the quantum mechanical rotor we could, however, not find a successful way employing QMC. On the other hand, the recursive numerical integration method works extremely well for this model and shows an at least exponentially fast error scaling.
\end{abstract}

Keywords: recursive numerical integration, quasi monte carlo, quantum mechanical rotor, anharmonic oscillator, lattice systems, low order couplings

\section{Introduction}

Markov Chain Monte Carlo (MCMC) is the method of choice for simulations of quantum field theories or systems in statistical physics. The advantage of MCMC is that it can be applied very generally to many physical models. It allows to compute expectation values of physical observables $\langle O\rangle$ with an error $\Delta$ which scales only as $\Delta \propto 1 / \sqrt{N}$, however, where $N$ is the number of samples. This error scaling law leads to a very large numerical effort if another significant digit in the accuracy of an observable is needed.

In quantum field theory, in particular quantum chromodynamics (QCD) our theory of the strong interaction between quarks and gluons - very significant progress has been achieved in the last years through improvements of the MCMC methods used; see, e.g., ref. [1] for an overview. But, even though lattice QCD simulations of the theory could be accelerated substantially, computations typically run several months or even years on state of the art supercomputers. In

Email addresses: andreas.ammon@desy.de (A. Ammon), genz@math.wsu.edu (A. Genz), tobias.hartung@kcl.ac.uk (T. Hartung), karl.jansen@desy.de (K. Jansen), leovey@math.hu-berlin.de (H. Leövey), julia.volmer@desy.de (J. Volmer) 
addition, none of those improvements have changed the error scaling of $1 / \sqrt{N}$. It will therefore be very demanding to obtain a significant improvement of the accuracy of physical observables in this field.

On the other hand, it is known that Quasi Monte Carlo (QMC) [2, 3] or recursive numerical integration [4, 5] methods show a much improved error scaling. For QMC methods this error scaling reads $\Delta \propto 1 / N^{\alpha}$ where $\alpha$ can reach values of $\alpha=1$ or even larger. For recursive numerical integration methods the error scaling can be even better and, in some cases, it is even faster than exponential, see also the discussion below.

Clearly, such QMC and recursive numerical integration methods could, thus, lead to a much enhanced accuracy of simulations. However, these methods have not been tried for generic quantum field theories, so far, and neither their applicability nor whether they lead to an improved error scaling is clear.

In [6, 7, 8, we initiated a test of QMC methods for the harmonic and anharmonic quantum mechanical oscillator discretized on a Euclidean time lattice; see the next section for an introduction to these systems. The results of these investigations have been very promising. For the harmonic oscillator, which is a Gaussian system, we found an error scaling of $\Delta \propto 1 / N$ which is optimal. Adding a non-Gaussian term in case of the anharmonic oscillator, we found $\alpha \approx 0.75$ which is not optimal but significantly better than the error scaling of MCMC methods. However, it needs to be mentioned that for certain system sizes, that is, large Euclidean times, the QMC method did not work particularly well for the anharmonic oscillator and no error scaling improvement could be established; cf., 7 for details.

However, QMC methods can also solve another problem: when using MCMC for the anharmonic oscillator, only samples in the vicinity of one minimum of the action are drawn and the probability to jump to another minimum is very small. This leads to a very large, in fact exponential, autocorrelation time. As demonstrated in refs. 6, 77, 8, with QMC using the harmonic approximation this problem is completely overcome. This is not surprising since QMC avoids long autocorrelation times essentially by construction. We stress that, with the new developments described in section 5.2 , the iterated numerical integration method allows to solve the anharmonic oscillator without any problem of autocorrelations, as well.

In this paper, we want to extend the work of refs. 6, 7, 8, in two directions. One direction is to apply recursive numerical integration methods for the anharmonic oscillator. As we will see below, this method does not suffer from the shortcomings of the QMC method for large Euclidean times. The other direction is the investigation of a topological quantum mechanical model, the quantum rotor. This model has some characteristic features that are also present for non-linear $\sigma$-models or gauge theories which are essential and most important models for describing elementary particle interactions; see, e.g., refs. 9, 10, 11 , for introductions to lattice field theories applied to particle theory. However, since the quantum rotor is a much simpler 1-dimensional quantum mechanical model, it is easier to treat numerically. In this way, it becomes possible to test in detail, whether QMC or recursive numerical integration methods work for such a model. Clearly, in case the application of one of the methods fails, it will become almost impossible to proceed with higher dimensional gauge theories. As we will discuss below, so far we have not been able to apply QMC successfully to the quantum rotor. On the other hand, recursive numerical integration 
methods turn out to be highly successful and show an extremely good error scaling behavior.

Another aspect investigated here is the rapid increase of the autocorrelation time for the quantum mechanical rotor when the continuum limit is approached. For the sake of illustrating this generic problem of MCMC methods, we will perform a calculation of the quantum rotor with the Metropolis algorithm. QMC and recursive numerical integration methods do not suffer from this problem and are, hence, also very advantageous in this respect. Since for the quantum mechanical rotor a particular MCMC method which also avoids the problem of very large autocorrelation times, the cluster algorithm, can be applied, we compare the recursive numerical integration method to this optimal MCMC algorithm to see whether a gain can still be found.

The paper is organized as follows. In the next section we introduce the models which are investigated throughout the paper. In section 3 we shortly summarize our (failed) attempts to solve the quantum rotor with QMC methods. In section 4 we introduce the recursive numerical integration method and discuss its theoretical basis. Finally, in section 5 we present our results and conclude in section 6 .

\section{Lattice systems: 1-dimensional models}

In this paper we investigate two different quantum mechanical models in Euclidean timø In order to evaluate the models numerically we will discretize time and solve the resulting high dimensional integrals numerically. In this section we start with some general considerations about calculating observables of lattice systems before we describe the two models we investigate, the topological oscillator and the anharmonic oscillator.

Calculating observables in discretized time. The coordinate $x(t) \in \mathbb{R}$ describes the trajectory of a particle in time. Classically, this path of a particle propagating from $x_{0}=x(0)$ to $x_{1}=x(T)$ during a time period $T$ is determined by the minimum of the action $\mathrm{S}(x)$ (with respect to $x(t)$ ),

$$
\mathrm{S}(x)=\int_{0}^{T} \mathcal{L}(x, t) d t \quad \in \mathbb{R},
$$

with the Lagrangian $\mathcal{L}(x, t)$ containing all necessary information about the model.

In order to obtain a numerically tractable and positive action while keeping the quantum mechanical observables real we have to define the trajectory $x(t)$ on a Euclidean, equidistant time lattice with lattice spacing $a$. Corresponding to this, we replace the following quantities by their discretized counterparts:

$$
\begin{array}{rr}
t \rightarrow t_{i}:=i \cdot a, \quad i \in\{0,1,2, \ldots, d-1\}, \\
x(t) \rightarrow x_{i}:=x\left(t_{i}\right), \quad x_{i} \in \mathbb{R},
\end{array}
$$

\footnotetext{
${ }^{1}$ Euclidean time is common practice in the standard lattice approach.
} 
with $T=a \cdot d$, such that

$$
\int_{0}^{T} d t \rightarrow a \sum_{i=0}^{d-1}, \quad \frac{d x}{d t} \rightarrow \nabla x_{i}=\frac{1}{a}\left(x_{i+1}-x_{i}\right) .
$$

We remark that the choice of the discretization of the derivative is not unique and alternative discretizations can lead to different error expansions of observables in terms of the lattice spacing in the continuum limit, $a \rightarrow 0$ and $T \rightarrow \infty$. We will use cyclic boundary conditions $x_{d}:=x_{d(\bmod d)}=x_{0}$ throughout this paper.

In a quantum mechanical system not only the classical path contributes to a given observable, but all possible paths have to be taken into account. Following Feynman's description, the quantum mechanical system is defined by the path integral

$$
\int_{D^{d}} \mathrm{e}^{-\mathrm{S}[x]} d x
$$

where $D$ is a domain in $\mathbb{R}$. In (3) the transformation to Euclidean time has been performed already. For a time discretized quantum mechanical system (3) is well-defined, although it may have a high dimension $d$, which could be 1000 or larger.

The expectation value $\langle O[x]\rangle$ of an observable $O[x]=O\left(x_{0}, x_{2}, \ldots, x_{d-1}\right)$ of a quantum mechanical model with a discretized action $S[x]$ can now be calculated using the path integral formalism

$$
\langle O[x]\rangle=\frac{\int_{D^{d}} O[x] \mathrm{e}^{-\mathrm{S}[x]} d x}{\int_{D^{d}} \mathrm{e}^{-\mathrm{S}[x]} d x} .
$$

We note that the formalism for treating quantum mechanical systems, as it is sketched out above, can be generalized to quantum field theories. Such quantum field theories are the actual basis for the theoretical investigation of elementary particle interactions.

Topological oscillator. As indicated previously, the first model we are going to consider in this article is the topological oscillator or quantum rotor which describes a particle with mass $M_{0}$ moving on a circle with radius $R$, and correspondingly, has a moment of inertia of $I=M_{0} R^{2}$. We investigate this particular model because it goes beyond the classical quantum mechanical oscillator (described later on) and already shows some characteristic features of non-linear $\sigma$-models and gauge theories which are of prime importance in particle physics.

The free coordinate of the system is the angle $\phi \in[-\pi, \pi)$, describing the position of the particle on a circle with radius $R$ around the origin. The system is described by the action

$$
S(\phi)=\int_{0}^{T} \frac{I}{2}\left(\frac{d \phi}{d t}\right)^{2} d t,
$$

and is obtained from the action of a free particle moving in two dimensions,

$$
\mathrm{S}(x)=\int_{0}^{T} \frac{M_{0}}{2}\left(\dot{x}(t)^{2}+\dot{y}(t)^{2}\right) d t \quad(x, y) \in \mathbb{R}^{2},
$$


and the transformation $x(t)=R \cos (\phi(t))$ and $y(t)=R \sin (\phi(t))$ respectively. The corresponding discretized action reads

$$
S[\phi]=\frac{I}{a} \sum_{i=0}^{d-1}\left(1-\cos \left(\phi_{i+1}-\phi_{i}\right)\right),
$$

where we use the cosine to describe the kinetic part of the action, as $\frac{1}{a^{2}}(1-$ $\left.\cos \left(\phi_{i+1}-\phi_{i}\right)\right)=\frac{1}{2}\left(\nabla \phi_{i}\right)^{2}+O\left(\left(\nabla \phi_{i}\right)^{4}\right)$ and hence corresponds to leading order to the naive lattice derivative (as in (2), right). In the following we will, if not stated otherwise, set the lattice spacing to $a=1$. This means, in particular, that the continuum limit is reached ${ }^{2}$ by $T=d \cdot a \rightarrow \infty$.

One characteristic quantity of the quantum mechanical rotor is the topological charge of the system. It describes the number of complete revolutions the rotor performs during a time period $T$

$$
Q(\phi)=\frac{1}{2 \pi} \int_{0}^{T}\left(\frac{d \phi}{d t}\right) d t \quad \in \mathbb{Z} .
$$

We use the discretized version

$$
Q[\phi]=\frac{1}{2 \pi} \sum_{i=0}^{d-1}\left(\phi_{i+1}-\phi_{i}\right) \bmod [-\pi, \pi) .
$$

As an observable, we will investigate the topological susceptibility

$$
\chi_{t}=\frac{\left\langle Q^{2}[\phi]\right\rangle}{T} \stackrel{T \rightarrow \infty}{\longrightarrow} \frac{1}{4 \pi^{2} I},
$$

where $\left\langle Q^{2}[\phi]\right\rangle$ is calculated according to 4 .

Other important observables of the system are the energy gaps which can be extracted from Euclidean correlation functions $\Gamma(j)$,

$$
\Gamma(j)=\frac{1}{d} \sum_{i=0}^{d-1} \phi_{i} \cdot \phi_{i+j}, \quad j \in\{0,1, \ldots, d / 2\} .
$$

It measures the correlation of angles at different lattice sites separated by a distance $j$. This correlation function has an exponential decay rate with the distance $j$,

$$
\Gamma(j) \tilde{\propto} \mathrm{e}^{-j \cdot \Delta E}, \quad j \gg 1 .
$$

From this the energy gap $\Delta E$ between the ground state and the first excited state and therefore also the correlation length $\xi$ can be determined,

$$
\xi=\frac{1}{\Delta E} \stackrel{T \rightarrow \infty}{\longrightarrow} 2 I .
$$

Other energy levels can be computed in a similar way.

\footnotetext{
${ }^{2}$ In principle, the physical extent of time lattice $T=d \cdot a$ should be kept constant and hence the continuum limit $a \rightarrow 0$ requires $d \rightarrow \infty$.
} 
Through the energy gap, a connection to the topological susceptibility can be established, as well; namely,

$$
\chi_{t}=\frac{\left\langle Q^{2}\right\rangle}{T}=\frac{1}{2 \pi^{2}} \Delta E .
$$

This, however, only holds in the continuum limit $a \rightarrow 0$ and $T \rightarrow \infty$.

Anharmonic oscillator. The second quantum mechanical system considered in this article is the harmonic oscillator which describes a particle with mass $M_{0}$ moving along a path $x \in \mathbb{R}$ in a potential proportional to $x^{2}$. Adding a $x^{4}$ term to the potential, the system is called the anharmonic oscillator. The Lagrangian is given by

$$
\mathcal{L}(x, t)=\frac{M_{0}}{2}\left(\frac{d x}{d t}\right)^{2}+\frac{\mu^{2}}{2} x^{2}+\lambda x^{4}
$$

where $\mu^{2}, \lambda \in \mathbb{R}$. In order to keep the action bounded from below, the coupling $\lambda$ has to be chosen positive. With $\lambda>0$ present, the constant $\mu^{2}$ can be chosen arbitrarily and for $\mu^{2}>0$ one finds a distorted harmonic potential while for $\mu^{2}<0$ a double well potential appears.

Using the discretization scheme mentioned in (1) and (2), the lattice action for the anharmonic oscillator becomes

$$
\mathrm{S}[x]=a \sum_{i=0}^{d-1} \frac{M_{0}}{2}\left(\nabla x_{i}\right)^{2}+\frac{\mu^{2}}{2} x_{i}^{2}+\lambda x_{i}^{4} .
$$

One type of characteristic observables of this system are powers of the position $x$ of the particle, i.e., $\langle x\rangle,\left\langle x^{2}\right\rangle,\left\langle x^{4}\right\rangle$, etc.

Another type of observable is again the energy gap which can be extracted from the correlator $\Gamma(j)$, as already described in eq. (7) and (8) for the topological oscillator. After exchanging the variables in eq. (7) by the ones of the anharmonic oscillator model a similar calculation has to be done here.

The harmonic and anharmonic quantum mechanical oscillators have been studied with QMC methods in [6, 7, 8].

\section{Remarks on Quasi-Monte Carlo attempts}

In this section, we would like to give an overview of our attempts to solve the topological oscillator with QMC methods utilizing (randomized) Sobol' sequences (cf., [12, 13] ${ }^{3}$. The section is intended for researchers who are familiar with QMC and may be skipped by non-experts. The addressed attempts were motivated by the positive results obtained for the (an)harmonic oscillator model (cf., e.g., 6] ). At the present state, however, we were not able to observe positive results with our selected QMC constructions for the topological oscillator model. In the following, we will summarize the sampling techniques that were tested in combination with (randomized) Sobol' sequences.

\footnotetext{
${ }^{3}$ These Sobol' sequences are low-discrepancy sequences, i.e., they are designed to be more uniformly distributed than pseudo-random numbers.
} 
Naïve Sampling. The naïve sampling here simply means that we used the (randomized) Sobol' points as an approximate uniform distribution and used these points directly to evaluate the (re-scaled) integrals according to

$$
\int_{[0,1]^{d}} f(x) d x \approx \frac{1}{N} \sum_{i=1}^{N} f\left(x_{i}\right)
$$

where the $x_{i}$ are the $d$-dimensional Sobol' points. As to be expected, it produced good results for small $\frac{I}{a}$ but loses accuracy and convergence speed as $\frac{I}{a}$ increases since most samples have little to no weight rendering them irrelevant. In fact, as $\frac{I}{a}$ grows significantly larger than $\frac{1}{2}$ we have not been able to observe any convergence in the range of sample sizes that are feasibly generatable.

Harmonic Sampling. Since we observed good results using the Sobol' sequences with the harmonic and anharmonic oscillator, it seemed reasonable to approximate

$$
\cos \left(\phi_{i}-\phi_{j}\right) \approx 1-\left(\phi_{i}-\phi_{j}\right)^{2},
$$

i.e., to draw just as in $[6$ and re-weight to account for the error made in drawing from this approximate distribution. Using the cumulative (standard) normal distribution $\Phi$, there are three obvious approaches to inverting $\Phi$ with range $[-\pi, \pi)$.

- Using $\mathbb{R}$ as a covering space of $[-\pi, \pi)$ yields the "inverse"

$$
(0,1) \rightarrow[-\pi, \pi) ; x \mapsto \Phi^{-1}(x) \operatorname{MOD} 2 \pi
$$

where

$$
\operatorname{MOD}: \mathbb{R} \rightarrow[-\pi, \pi) ; x \mapsto x-\operatorname{round}\left(\frac{x}{2 \pi}\right) 2 \pi=x-\left\lfloor\frac{x}{2 \pi}+\frac{1}{2}\right\rfloor 2 \pi .
$$

- Use the complete normal distribution and dismiss points not in $[-\pi, \pi]^{d}$. (Note that this approach destroys the property of uniformity of the Sobol' sequence by rejecting some of the points.)

- Restrict to $[-\pi, \pi)$-slice of the normal distribution, i.e., invert

$$
\tilde{\Phi}(x):=\frac{\Phi(x)-\Phi(-\pi)}{2 \Phi(\pi)-1} .
$$

Qualitatively, all three approaches yield the same result; our quadrature was highly instable yielding seemingly random numbers and we have not been able to stabilize them. We think this is due to an inherent under-representation of samples in the region where $\left|\phi_{i+1}-\phi_{i}\right|$ is close to $2 \pi$. These samples have a large weight but the chance of drawing them is slim. Hence, hitting these regions a little more often can make a significant difference which we observe as an instability of our results. 
Inversive Samplings. Inversive samplings (cf., 14 chapter II.2) go one step further than the harmonic sampling by choosing better approximations than the harmonic one. However, we still have the constraint that we need to be able to effectively draw from the distribution. In this case, we are looking for an expression of the form

$$
\exp (-S[\phi]) \approx \prod_{j=0}^{d-1} p_{j}\left(\phi_{j}\right)
$$

In fact, the two main examples we tested were of the form

$$
\exp (-S[\phi]) \approx \prod_{j=0}^{d-1} p\left(\phi_{j}\right)
$$

where $p$ is a polynomial or a step-function. The step-function has the advantage that we can draw with very little effort. However, QMC does not like discontinuous integrands, i.e., we expect to lose convergence speed. Choosing a polynomial $p$ does not have that draw-back but generating samples is a lot harder since it involves numerically inverting the cumulative distribution function defined by the polynomial used as a density.

Our results are satisfactory in the sense that we did not observe an increase in autocorrelation time going to small lattice spacings (in fact, we would be highly surprised if that happened since we are not using a random walk to generate samples) but we have not been able to improve over standard Monte Carlo methods where they are applicable, that is, to obtain an error scaling better than $O\left(N^{-0.5}\right)$ where $N$ is the sample size.

Sampling - Importance Resampling. SIR (cf., [15, 16, 17]) is a closely related concept where a pool of sample points is generated (here, using (randomized) Sobol' sequences) and stored. Samples are then to be drawn from the discrete distribution of points in the pool with respect to their weight in the target distribution (cf., e.g., 14 chapter III.2). It worked fairly well for small sample sizes but, trying to go to large numbers of samples, the pool must increase as well in order to keep the systematic error of the changed distribution small rendering this method too memory demanding to be viable for high accuracy calculations (an error analysis of QMC based SIR methods can be found in [18]).

Envelope Inversive Rejective Samplings. An approach to draw directly from the target distribution but with the help of inversive sampling starts by choosing an approximation $\tilde{S}[\phi]$ of $S[\phi]$ such that

$$
e^{-S[\phi]} \leq e^{-\tilde{S}[\phi]}
$$

We call such an inversive sampling an Envelope Inversive Sampling. Drawing from the envelope, we may now use a rejection step (cf., [19]) to ensure drawing from the target distribution in the usual Monte Carlo manner.

Our results are comparable to the Inversive sampling results, that is, no increased auto-correlation for small lattice spacings but also no accelerated convergence with respect to standard Monte-Carlo methods. 
Smoothed Envelope Inversive Rejective Samplings. Here, the idea is that we might obtain an edge starting from the envelope inversive rejective sampling by smoothing the integrand; viz., the rejective step is a step-function in the integrand which behaves poorly with QMC and, hence, may yield better results if subjected to a smoothing operation. The method of Smoothed Rejection can be found in 20 and effectively replaces the step-function describing the rejection step by a continuous function and additional re-weighting. However, we could not observe any improvement over the envelope inversive rejective sampling.

\section{The method of recursive numerical integration}

In this section, we consider the method of recursive numerical integration (see 4, 5] and references therein) also sometimes called the method of iterated numerical integration, for the approximation of the integration problems in 1dimensional lattice theory stated in section 2, The main idea of this method is based on the fact that if the integrand at hand can be described as the product of low-dimensional functions, each one describing the interactions or couplings between few consecutive objects, then we can write the final highdimensional integration problem as the iteration of coupled low-dimensional integrals. At this point a quadrature rule for low-dimensional integration has to be chosen in order to carry out the successive approximation of the underlying low-dimensional integrals. In the following, we will consider the case of simple 1-neighboring couplings for simplicity. For a description of the general case with several neighboring couplings or branchings, we refer again to $\underline{5}$. Thus, we have an integrand function of the form

$$
f(x)=\prod_{i=0}^{d-1} f_{i}\left(x_{i}, x_{(i+1)(d)}\right)
$$

and we would like to approximate

$$
I=\int_{D^{d}} \prod_{i=0}^{d-1} f_{i}\left(x_{i}, x_{(i+1)(d)}\right) d \mathbf{x},
$$

where the notation $().(d)$ means to take the argument modulo $d$, and $D \subset \mathbb{R}$ is a 1-dimensional domain. Thus, by the Fubini-Tonelli theorem, assuming that the desired integral value exists, we can write the problem equivalently as

$$
\begin{aligned}
I= & \int_{D}\left(\int_{D} f_{0}\left(x_{0}, x_{1}\right) \ldots\right. \\
& \ldots\left(\int_{D} f_{d-3}\left(x_{d-3}, x_{d-2}\right)\left(\int_{D} f_{d-2}\left(x_{d-2}, x_{d-1}\right) f_{d-1}\left(x_{d-1}, x_{0}\right) d x_{d-1}\right) d x_{d-2}\right) \ldots \\
& \left.\ldots d x_{1}\right) d x_{0} .
\end{aligned}
$$

Furthermore, we can consider scalings $c_{0}, \ldots, c_{d-1}>0$, and define $I^{\star}:=$ $\left(\prod_{i=0}^{n-1} \frac{1}{c_{i}}\right) I$, such that

$$
\begin{aligned}
I^{\star}= & \int_{D}\left(\int_{D} \frac{f_{0}\left(x_{0}, x_{1}\right)}{c_{0}} \ldots\right. \\
& \ldots\left(\int_{D} \frac{f_{d-3}\left(x_{d-3}, x_{d-2}\right)}{c_{d-3}}\left(\int_{D} \frac{f_{d-2}\left(x_{d-2}, x_{d-1}\right) f_{d-1}\left(x_{d-1}, x_{0}\right)}{c_{d-2} c_{d-1}} d x_{d-1}\right) d x_{d-2}\right) \ldots \\
& \left.\ldots d x_{1}\right) d x_{0} .
\end{aligned}
$$


Usually the quantities $c_{0}, \ldots, c_{d-1}$ will have to be chosen adaptively as they are used in order to avoid under/over-flows in the recursive method for high dimensions due to limited machine accuracy. By selecting an adequate 1-dimensional quadrature rule $Q$ with $m$ points and weights for approximating the underlying integration problems over $D$ in each iteration, we can write the iteration method in recipe form as

1. Fix, if possible, a quadrature rule with $m$ points $x^{1}, \ldots, x^{m}$ and weights $w_{1}, \ldots, w_{m}$ that works well for one dimensional integrals of type

$$
\int_{D} f_{i-1}\left(\theta_{1}, z\right) f_{i}\left(z, \theta_{2}\right) d z, \quad \text { for } 1 \leq i \leq d-1, \theta_{1}, \theta_{2} \in D .
$$

2. Use the quadrature in 1 . to estimate

$$
\begin{aligned}
F_{d-1}\left(x_{d-2}, x_{0}\right) & :=\frac{1}{c_{d-2} c_{d-1}} \int_{D} f_{d-2}\left(x_{d-2}, x_{d-1}\right) f_{d-1}\left(x_{d-1}, x_{0}\right) d x_{d-1} \\
& \approx \frac{1}{c_{d-2} c_{d-1}} \sum_{j=1}^{m} w_{j} f_{d-2}\left(x_{d-2}, x^{j}\right) f_{d-1}\left(x^{j}, x_{0}\right)
\end{aligned}
$$

over the grid of points $\left\{x^{1}, \ldots, x^{m}\right\} \times\left\{x^{1}, \ldots, x^{m}\right\} \subset D^{2}$. By defining the transfer $m \times m$ matrix

$$
M_{i}(k, l):=f_{i}\left(x^{k}, x^{l}\right), \quad 1 \leq k, l \leq m, \quad 0 \leq i \leq d-1,
$$

we can write in matrix form

$$
\left[F_{d-1}\left(x_{d-2}^{i}, x_{0}^{j}\right)\right]_{1 \leq i, j \leq m} \approx \frac{1}{c_{d-2} c_{d-1}} M_{d-2} \operatorname{diag}\left(\left(w_{1}, \ldots, w_{m}\right)\right) M_{d-1},
$$

where

$$
\operatorname{diag}\left(\left(w_{1}, \ldots, w_{m}\right)\right):=\left(\begin{array}{cccc}
w_{1} & 0 & \cdots & 0 \\
0 & w_{2} & \cdots & 0 \\
\vdots & \vdots & \ddots & \vdots \\
0 & 0 & \cdots & w_{m}
\end{array}\right)
$$

3. For $i=d-2, d-3, \ldots, 2$ approximate iteratively

$$
\begin{aligned}
F_{i}\left(x_{i-1}, x_{0}\right) & =\frac{1}{c_{i-1}} \int_{D} f_{i-1}\left(x_{i-1}, x_{i}\right) F_{i+1}\left(x_{i}, x_{0}\right) d x_{i} \\
& \approx \frac{1}{c_{i-1}} \sum_{j=1}^{m} w_{j} f_{i-1}\left(x_{i-1}, x^{j}\right) F_{i+1}\left(x^{j}, x_{0}\right)
\end{aligned}
$$

over the grid of points $\left\{x^{1}, \ldots, x^{m}\right\} \times\left\{x^{1}, \ldots, x^{m}\right\} \subset D^{2}$. Thus, the result of step 3. over $\left\{x^{1}, \ldots, x^{m}\right\} \times\left\{x^{1}, \ldots, x^{m}\right\}$ can be written as the matrix products

$$
\left[F_{2}\left(x_{1}^{i}, x_{0}^{j}\right)\right]_{1 \leq i, j \leq m} \approx\left(\prod_{i=1}^{d-2} \frac{1}{c_{i}} M_{i} \operatorname{diag}\left(\left(w_{1}, \ldots, w_{m}\right)\right)\right) \frac{1}{c_{d-1}} M_{d-1},
$$

with the notation for matrix products $\prod_{i=1}^{d-2} B_{i}:=B_{1} B_{2} \ldots B_{d-2}$. 
4. Estimate with the $m$-point quadrature in 1 . the function

$$
F_{1}\left(x_{0}, x_{0}\right)=\int_{D} \frac{f_{0}\left(x_{1}, x_{0}\right) F_{2}\left(x_{1}, x_{0}\right)}{c_{0}} d x_{1} .
$$

over the grid $\left\{x_{0}^{1}, \ldots, x_{0}^{m}\right\} \subset D$. Finally, estimate with the $m$-point quadrature in 1 . the function

$$
I^{\star}=\int_{D} F_{1}\left(x_{0}, x_{0}\right) d x_{0} .
$$

The result of this step can be written in matrix operations as

$$
\begin{aligned}
I^{\star} & \approx \operatorname{Tr}\left(\operatorname{diag}\left(\left(w_{1}, \ldots, w_{m}\right)\right)\left(\prod_{i=0}^{d-2} \frac{1}{c_{i}} M_{i} \operatorname{diag}\left(\left(w_{1}, \ldots, w_{m}\right)\right)\right) \frac{1}{c_{d-1}} M_{d-1}\right) \\
& =\operatorname{Tr}\left(\prod_{i=0}^{d-1} \frac{1}{c_{i}} M_{i} \operatorname{diag}\left(\left(w_{1}, \ldots, w_{m}\right)\right)\right) .
\end{aligned}
$$

As mentioned in the previous section we are mainly interested in considering two types of integrands for high-dimensional integration. The first type of integrand is given as the weight of the Boltzmann distribution. In this case we usually obtain that the functions $f_{i}, 0 \leq i \leq d-1$, satisfy $f_{0}=f_{1}=\cdots=f_{d-1}$ due to isotropic conditions of the model. Thus, this case yields $M_{0}=M_{1}=$ $\cdots=M_{d-1}=: M$ and we can choose $c_{0}=c_{1}=\cdots=c_{d-1}=: c$. Hence, the identity above reduces to

$$
I^{\star}=\operatorname{Tr}\left(\left(\frac{1}{c} \operatorname{Mdiag}\left(\left(w_{1}, \ldots, w_{m}\right)\right)\right)^{d}\right) .
$$

Note that the similarity relation

$$
\operatorname{Mdiag}\left(\left(w_{1}, \ldots, w_{m}\right)\right) \sim \operatorname{diag}\left(\left(\sqrt{w_{1}}, \ldots, \sqrt{w_{m}}\right)\right) \operatorname{Mdiag}\left(\left(\sqrt{w_{1}}, \ldots, \sqrt{w_{m}}\right)\right),
$$

holds, where for $m \times m$ matrices $A, B$ we say that $A \sim B$ if and only if $A=C B C^{-1}$, for an invertible matrix $C$. The resulting matrix on the right hand side of the relation above is symmetric and efficient algorithms are known for its diagonalization (cf., 21]). By use of the eigenvalue decomposition of a diagonalizable matrix $A$, we can write $A^{d}=C D^{d} C^{-1}$ where $D$ is the diagonal matrix of eigenvalues, and $C$ is the corresponding invertible matrix of eigenvectors. Because the trace of a diagonalizable matrix equals the sum of its eigenvalues, to calculate the trace of $A^{d}$ we only need to calculate the eigenvalues of $A$, raise them to the power of $d$, and finally sum them up. It is also worth to mention that for particular applications, recursive-multiplication for calculation of $\left(\frac{1}{c} M \operatorname{diag}\left(\left(w_{1}, \ldots, w_{m}\right)\right)\right)^{d}$ may be also a competitive method. The second type of integrand is given by the Boltzmann weight times an observable function. For simple observable functions usually considered in lattice theory, similar gains over direct matrix-matrix multiplications can be obtained by condensing a sequence of matrix-matrix multiplications into a power form. Particular examples and the corresponding implementations will be discussed in detail in the following section. 


\section{Numerical experiments}

In this section, we will investigate the applicability of the method of recursive numerical integration to the topological rotor and the anharmonic oscillator, as described in section 2. In particular, we will compare the calculations of recursive numerical integration methods with standard MCMC methods applied to lattice systems [11, 22 and also with improved methods, i.e., the cluster algorithm [23] and randomized QMC methods for the anharmonic oscillator [7.

\subsection{Topological oscillator}

For the sake of illustrating the difficulties that can appear in standard MCMC calculations, we will perform initial computations with the Metropolis algorithm 24]. This algorithm, although very generally applicable, is often not the optimal choice to simulate a model and is usually replaced by a more suitable technique. However, here we use the Metropolis algorithm to demonstrate the generic difficulty of many MCMC methods that towards the continuum limit the autocorrelation time grows rapidly. At fixed number of samples, this leads to very large errors when the lattice spacing is reduced and, correspondingly, to highly increasing computational costs of the simulations. Throughout the discussion below, we use a moment of inertia of $I=0.25$ for the quantum mechanical topological rotor.

Besides promising a much improved error scaling, recursive numerical integration methods avoid this explosion of the autocorrelation time and are therefore highly superior to MCMC techniques. Nevertheless, for special situations there exist MCMC methods which also avoid the increase in autocorrelation time. For the topological rotor investigated here, cluster algorithms [23] can be applied which exhibit a basically constant behavior of the autocorrelation time with respect to the lattice spacing while still showing the $1 / \sqrt{N}$ error scaling behavior of MCMC methods. Hence, we will explore how the recursive numerical integration technique compares to an optimal MCMC method such as the cluster algorithm and whether a gain can still be found. Since for the quantum mechanical topological rotor we could not find a satisfactory realization of QMC methods to solve the system, we will not discuss this approach in this section.

\subsubsection{MCMC method}

A basic algorithm of the MCMC class is the Metropolis algorithm [24] which, as discussed above, will be used for illustration purposes only. The Metropolis algorithm uses importance sampling as described in section 3 to create samples of a given system on which observables $O$ can be calculated. These observables are then averaged over all samples to give the expectation value $\langle O\rangle$ with an error $\Delta\langle O\rangle \propto 1 / \sqrt{N}$, where $N$ is the number of samples. The problem of the Metropolis algorithm - and MCMC methods in general - is that the samples are not all independent of each other but correlated. This correlation is measured through the autocorrelation time which is a property of the employed algorithm and, if the so-called integrated autocorrelation time is taken, also of the considered observable.

The behavior of the error and the integrated autocorrelation time of the topological susceptibility when applying the Metropolis algorithm as a function of the lattice spacing $a$ is shown in figure 1. We have chosen the topological susceptibility as an observable, since it is very sensitive to correlations between 
$\Delta \chi_{\text {top }}$ vs. lattice spacing

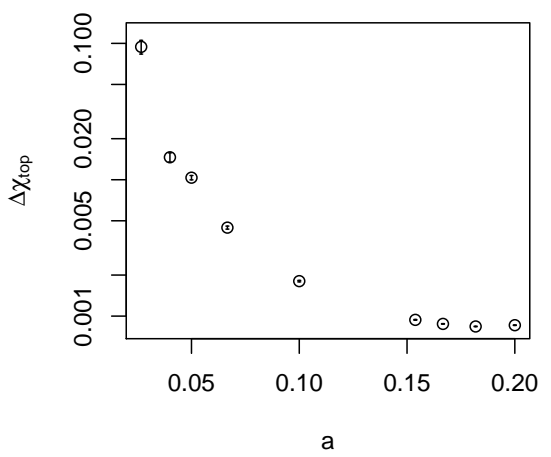

$\tau_{\text {int }}$ vs. lattice spacing

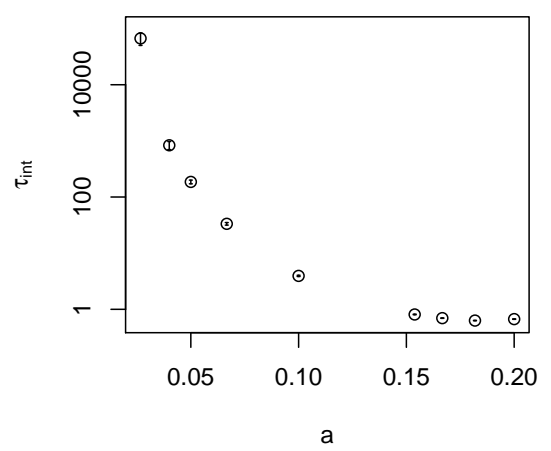

Figure 1: Logarithmic error and logarithmic integrated autocorrelation time behavior of the Metropolis algorithm for fixed number of samples $\left(N=10^{5}\right)$ dependent on the lattice spacing $a$. Left: Error of the topological susceptibility $\Delta \chi_{\text {top }}$, right: the corresponding integrated autocorrelation time $\tau_{\text {int }}$.

samples and often shows the largest integrated autocorrelation time in a simulation. It is also for this reason that the quantum mechanical topological rotor, where topological effects can be studied, is a good test case to compare MCMC and QMC or recursive numerical integration methods.

We remark that for all values of the lattice spacing shown in fig. 1 a fixed number of samples $\left(N=10^{5}\right)$ has been used. In addition, for the error calculation the integrated autocorrelation time has been fully taken into account. As can be observed in fig. 1, the integrated autocorrelation time $\tau_{\text {int }}$ (right plot) and therefore the error of the topological susceptibility $\Delta \chi_{\text {top }}$ (left plot) are rapidly growing with decreasing lattice spacing $a$. This leads to highly increased computational costs.

For systems in statistical physics or quantum field theory the continuum limit is reached by approaching a critical point. Since the rapid increase of the autocorrelation time in this limit is generic for many MCMC algorithms, this constitutes a most severe problem when higher dimensional systems are explored.

For the explicit model considered here, there exists a cluster algorithm [23] which avoids the problem of an increasing autocorrelation time for shrinking lattice spacings. Figure 2 shows the behavior of the autocorrelation time (right plot) and the error of the topological susceptibility (left plot) for the cluster algorithm as a function of $a$. As can be seen for the cluster algorithm, there is even a tendency that both, the error and the autocorrelation time, shrink for smaller $a$.

To compare the two algorithms directly, figure 3 shows the observable $\chi_{\text {top }}$ dependent on $a$ for the Metropolis and the cluster algorithms. For larger values of $a$ the error from both algorithms are similar. However, for small values of $a$ the error of the Metropolis algorithm becomes so large that it would be very demanding to reach an accurate result below a certain value of the lattice spacing, thus making a continuum extrapolation of $\chi_{\text {top }}$ rather difficult. On the 
$\Delta \chi_{\text {top }}$ vs. lattice spacing

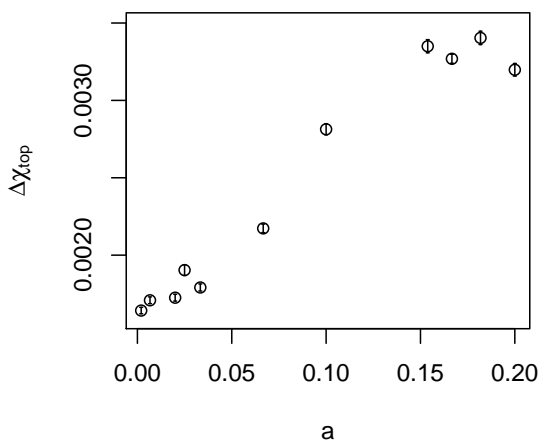

$\tau_{\text {int }}$ vs. lattice spacing

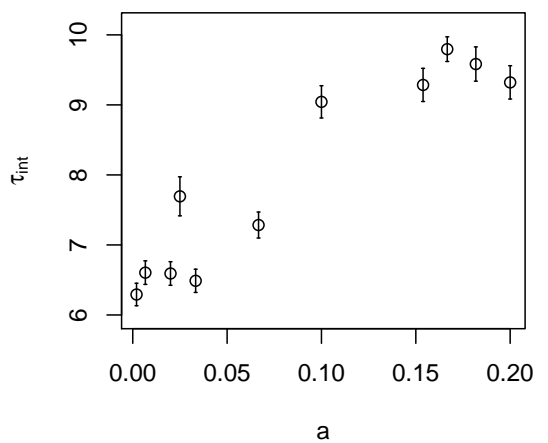

Figure 2: Error and integrated autocorrelation time behavior of the cluster algorithm for fixed number of samples $\left(N=10^{5}\right)$ dependent on the lattice spacing a. Left: Error of the topological susceptibility $\Delta \chi_{\text {top }}$, right: the corresponding integrated autocorrelation time $\tau_{\text {int }}$.

other hand, for the cluster algorithm the error stays practically constant; hence, allowing us to reach very small values of the lattice spacing giving a much better control of the continuum limit.

We stress again that the discussion above is only intended for an illustration of the generic behavior of MCMC methods and to demonstrate the problem of an increasing autocorrelation time when approaching the continuum limit for certain classes of MCMC algorithms. As the examples show, MCMC methods often have to face the difficulty of very large autocorrelation times which would be absent for QMC or recursive numerical integration methods. In the model considered here an optimal algorithm can be used, the cluster algorithm. It will therefore be interesting to see, whether the recursive numerical integration method is still advantageous even for cases when highly improved MCMC techniques can be employed. Although not relevant for this paper, we would like to mention that cluster algorithms are not applicable to gauge theories, so far.

\subsubsection{Recursive Gaussian quadrature}

In order to demonstrate the precision that can be reached with the recursive numerical integration method even at very small values of the lattice spacing, we will first discuss some numerical results that we have obtained with this approach. To this end, we have implemented the method of recursive numerical integration described in section 4, using Gauss-Legendre mesh points (abscissae), and applied it to the topological oscillator. Fixing the number of integration points to $m=120$ and the value of the moment of inertia $I=0.25$, we calculated the topological susceptibility $\chi_{\text {top }}$, the energy gap $\Delta E$ and the ratio of both observables $\frac{\chi_{\text {top }}}{\Delta E}$ as a function of the lattice spacing; cf., fig. 4 .

For the employed value of $I=0.25$, there are theoretical predictions for the observables we consider here, see section 2, In particular, in the continuum limit we should find for the topological susceptibility $\chi_{\text {top }} \rightarrow \frac{1}{4 \pi^{2} I}=\frac{1}{\pi^{2}}$, for the energy gap $\Delta E \rightarrow \frac{1}{2 I}=2$, and for the ratio $\frac{\chi_{\text {top }}}{\Delta E} \rightarrow \frac{1}{2 \pi^{2}}$. 


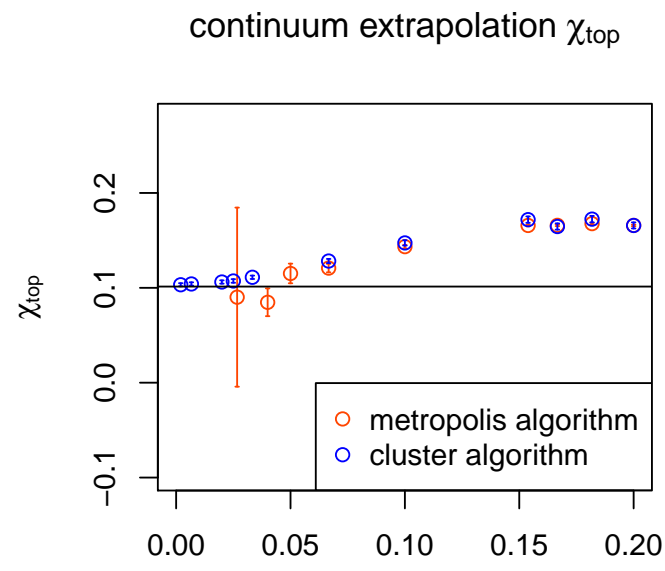

a

Figure 3: Continuum extrapolation of the topological susceptibility $\chi_{\text {top }}$ calculated by the Metropolis and the cluster algorithms for a fixed number of samples $\left(N=10^{5}\right)$.

In figure 4, we show our results obtained with recursive Gauss-Legendre quadrature with the expected continuum values, as given above, subtracted. The graphs nicely show that for all observables the results converge to zero; thus, being fully consistent with the theoretical expectations. Note that in the left column of fig. 4 we use a linear scale for the observables considered while in the right column a logarithmic scale is used which demonstrates the high precision we can reach with recursive Gauss-Legendre quadrature. Furthermore, note that the ratio $\frac{\chi_{\text {top }}}{\Delta E}$ has a peak at higher values of $a$, due to peculiar lattice artifacts, which is consistent with previous studies of this model [25].

Let us now turn to the interesting question of the error scaling for the recursive integration method. The behavior of the error for the topological susceptibility is shown in figure 5 for a fixed lattice spacing $a=0.4$ as a function of the number of integration points $m$. Note that we use a logarithmic scale for plotting the error of the topological susceptibility. We define the error by the difference of $\chi_{\text {top }}$ obtained for $m=560$ and $\chi_{\text {top }}$ computed at the given number of integration points $m \leq 480$. For $220 \leq m \leq 480$ we fit an exponential function of the error in $m$ which appears as a straight line in fig. 5 where a logarithmic scale is used. The good agreement between the data and this exponential fit suggests that asymptotically the error scales down at least exponentially fast. Recall that the error behavior for Gauss-Legendre quadrature with $m$ points for infinitely differentiable functions scales like $O\left(\frac{1}{(2 m) !}\right) \sim O\left(\frac{e^{2 m}}{\sqrt{2 \pi 2 m}(2 m)^{2 m}}\right)$, where the latter relation holds due to Stirling's approximation formula.

It is clear that with asymptotic exponential (or even better) error scaling the numerical recursive integration method will outperform any algorithm that shows an algebraic error scaling, in particular the $1 / \sqrt{N}$ behavior of MCMC algorithms. Still, it is an interesting question whether for small, more practical 


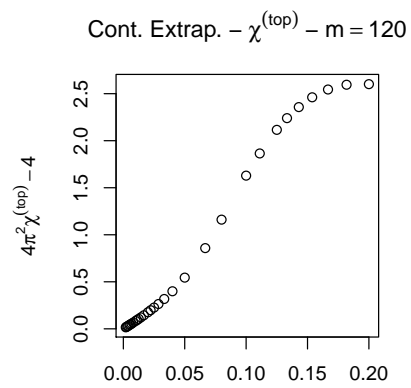

a

Cont. Extrap. $-\Delta E-m=120$

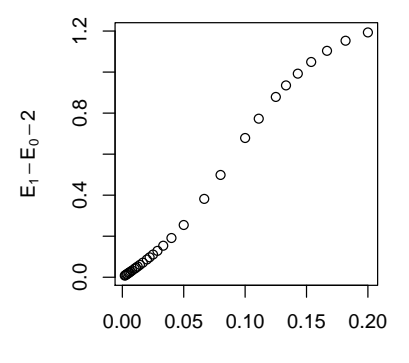

Cont. Extrap. - Ratio $-\mathrm{m}=120$

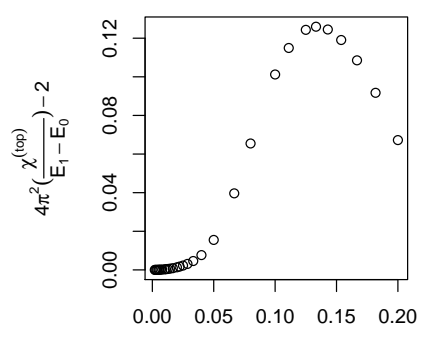

a
Cont. Extrap. $-\chi^{(\text {top })}-m=120$

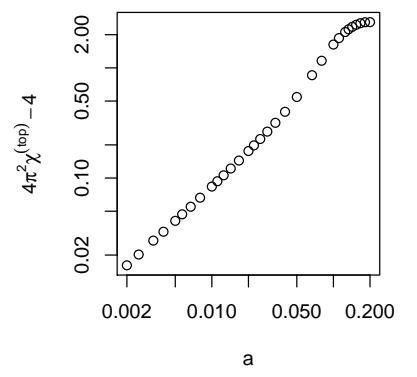

Cont. Extrap. $-\Delta \mathrm{E}-\mathrm{m}=120$

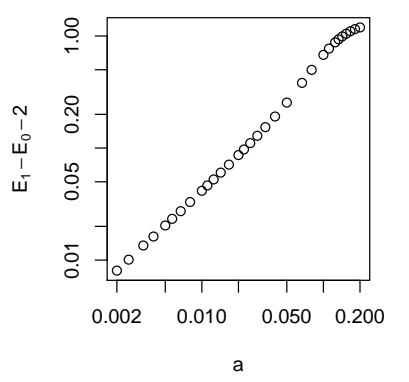

Cont. Extrap. - Ratio $-\mathrm{m}=120$

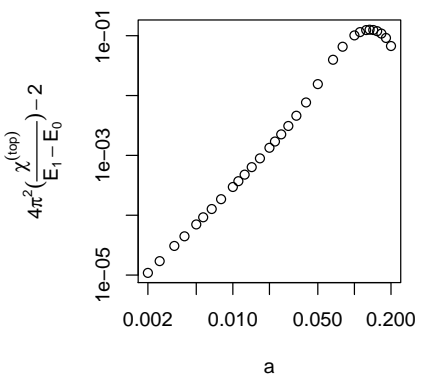

Figure 4: Gaussian quadrature - continuum extrapolation of three different observables, the topological susceptibility $\chi_{\text {top }}$ (top row), the energy gap $\Delta E=$ $E_{1}-E_{0}$ (middle row) and the ratio $\frac{\chi_{\text {top }}}{\Delta E}$ (bottom row) with $m=120$ integration points. Each observable is presented with a linear axis scale (left column) and a logarithmic axis scale (right column). For all results shown, we have subtracted the theoretical value of the given observable in the continuum such that they should converge to zero in the continuum limit.

values of $N$ (or $m$ ) a gain can be obtained from the recursive integration method.

We, therefore, measured the run-time of the Gauss and the cluster algorithms needed to obtain a given error of the topological susceptibility. For these measurements we ran both algorithms with the same input parameters (we used here a lattice spacing of $a=0.1$ ) on the same stand-alone computer. We then varied the number of samples and integration points for the cluster and recur- 


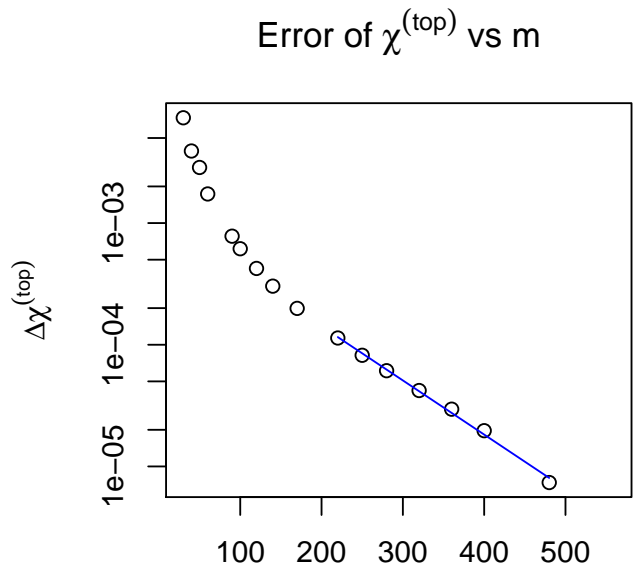

$\mathrm{m}$

Figure 5: Gaussian quadrature - error behavior of $\chi_{\text {top }}$ in dependence of the number of points $m$ used in the integration and with fixed lattice constant $a=0.4$. Note that we use a logarithmic scale to exhibit the error. The blue line is an exponential fit of the error for $220 \leq m \leq 480$ which appears - through the use of a logarithmic scale - as a straight line for a function of $m$. The consistency of the data with the linear behavior suggest that the error scales down at least exponentially fast.

sive Gauss-Legendre quadrature, respectively, and measured the run-time. We repeated each measurement ten times and averaged over the measured times to get an error estimate of the run-time. This error originates from the number and kind of other processes that are running on the machine at a given time and is noticeable for small run-times. For the cluster algorithm, in addition, the size and distributions of the generated clusters can vary leading to different run-times of the algorithm.

Being an MCMC method, for the cluster algorithm the procedure of repeating all runs ten times allows us to also determine the error on $\Delta \chi_{\text {top }}$, i.e., the error of the error. The recursive Gauss-Legendre quadrature, on the other hand, is purely deterministic and, therefore, gives the same result for $\chi_{\text {top }}$ without any error every time we run it. Since for this test we have used different parameters, we have also chosen a different gauge value of $m=400$ and calculate the error for $\chi_{i}(m<400)$ via the difference to this value, $\Delta \chi_{i}=\left|\chi_{i}-\chi_{i}(m=400)\right|$. The error of this error is neglected here.

In figure 6, the run-time $t$ needed to achieve a given error of the topological susceptibility is shown for both algorithms. Note that the graph is shown in a double logarithmic scale. The cluster algorithm shows the for MCMC methods typical $1 / \sqrt{t}$ behavior, indicated by the red line. The recursive Gauss-Legendre quadrature has a much steeper negative slope, such that the calculation to reduce the error by a specific amount is substantially faster than using the cluster algorithm. Additionally, even for small run-times the recursive Gauss-Legendre quadrature shows a much reduced error already. Therefore, the recursive Gauss- 


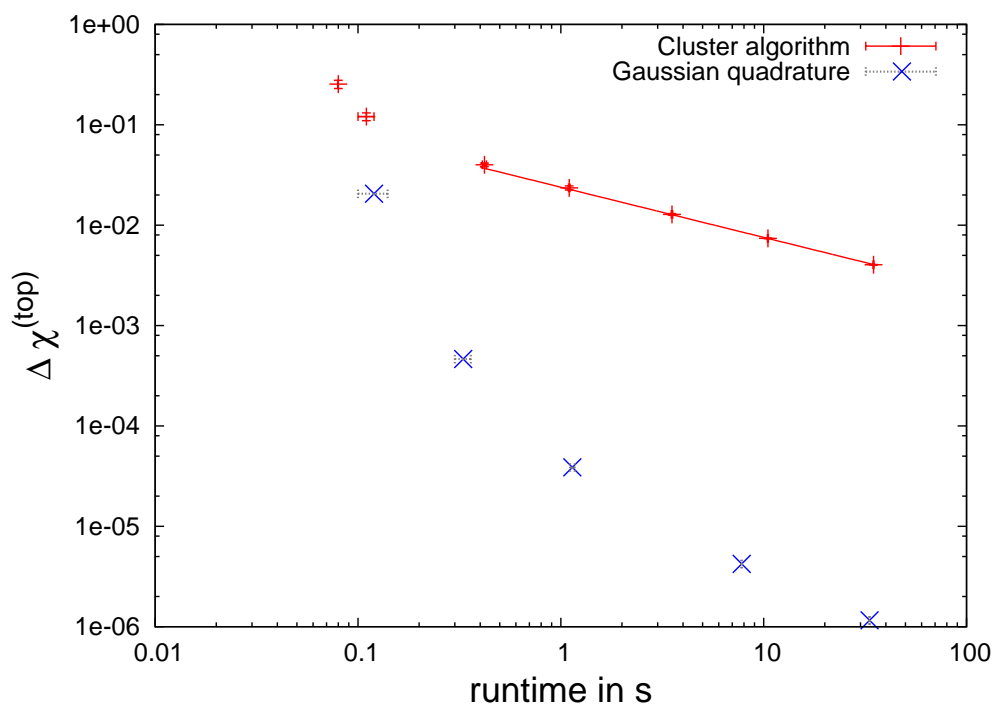

Figure 6: Comparison of Cluster algorithm and recursive Gauss-Legendre quadrature. We show the run-time $t$ in seconds needed to get a given error on the topological susceptibility with fixed lattice spacing $a=0.1$ on a standalone computer. Note that the graph is plotted in a double logarithmic scale. We used $N=10^{2} \ldots 10^{6}$ samples for the cluster algorithm and $m=10 \ldots 300$ integration points for the Gauss algorithm. To achieve the error of the error the numerical experiment has been repeated ten times for a fixed set of parameters. The run-time error of both algorithms results from different execution times of the program due to state of the computer when the program was executed. For the cluster algorithm the error in the run-time is also determined from different distributions of clusters generated. The red line shows the expected $1 / \sqrt{t}$ behavior of the cluster algorithm as a MCMC method.

Legendre quadrature is not only superior in the asymptotic regime at large runtimes where it shows a much improved error scaling but is also advantageous when only small run-times are employed. This makes it a promising approach if one thinks of systems in higher dimensions where only short run-times can be afforded.

\subsection{Anharmonic oscillator}

Applications of QMC methods to the anharmonic oscillator model and comparison with MCMC techniques have been studied in [6, 7, 8. In particular, the superiority of randomized QMC techniques applied to the anharmonic oscillator model has been observed for small time periods $T \leq 1.5$ independently of the size of lattice spacing $a$. Here we investigate whether the recursive integration method can also be successfully applied to this model and whether improvements over randomized QMC can be observed. To this end, we carry out tests by choosing different pairs of problem dimension and spacing $(d, a)$. 
The time period $T$ associated to a pair $(d, a)$ is given by $T=d a$. The choice of the pairs $(d, a)$ has been done with the aim to show that the anharmonic oscillator model can be approximated satisfactorily with a particular recursive numerical integration method. The observables investigated are $\left\langle x^{2}\right\rangle$ and $\left\langle x^{4}\right\rangle$. The rest of the parameters of the model have been fixed for the experiments to $M_{0}=0.5, \mu^{2}=-16.0$, and $\lambda=1.0$. We perform recursive one-dimensional integration based on Gauss-Legendre quadrature. To this end, we first re-scale the integration variables and re-arrange the terms in the action such that (using the same notation as in section 4 we end up with a transition function

$$
f_{i}\left(x_{i}, x_{i+1}\right)=e^{-\left(x_{i}-x_{i+1}\right)^{2}-\frac{1}{2}\left(\frac{\mu^{2} a^{2}}{M_{0}}\left(x_{i}^{2}+x_{i+1}^{2}\right)+\frac{4 \lambda a^{3}}{M_{0}^{2}}\left(x_{i}^{4}+x_{i+1}^{4}\right)\right)} .
$$

The quartic negative term $-\frac{4 \lambda a^{3}}{M_{0}^{2}} x_{i}^{4}$ dominates the quadratic positive term $-\frac{\mu^{2} a^{2}}{M_{0}} x_{i}^{2}$ when $\left|x_{i}\right| \geq \sqrt{\frac{M_{0}\left|\mu^{2}\right|}{4 \lambda a}}$, for $i=1, \ldots, d$. Outside of the region $[-$ $\sqrt{\frac{M_{0}\left|\mu^{2}\right|}{4 \lambda a}}, \sqrt{\left.\frac{M_{0}\left|\mu^{2}\right|}{4 \lambda a}\right]^{2}}$, the term inside the exponential in the transition function $f_{i}\left(x_{i}, x_{i+1}\right)$ remains always negative, and decays mainly with a quartic rate that adds to the quadratic coupling $-\left(x_{i}-x_{i+1}\right)^{2}$. Therefore the marginal tails of this two-dimensional transition function decay faster than the marginal tails of a bivariate normal, and this fact can be used to select a region of main importance for the one-dimensional parametric integration problems described in (10). Thus, we additionally search to ensure that the decay obtained by the dominant quartic term overshadows the contribution of the remaining coupling quadratic term $-\left(x_{i}-x_{i+1}\right)^{2}$ in the action. As an heuristic, we then chose the main importance region for integration to be of the form $\left[-p \sqrt{\frac{M_{0}\left|\mu^{2}\right|}{4 \lambda a}}, p \sqrt{\frac{M_{0}\left|\mu^{2}\right|}{4 \lambda a}}\right]$, for a number $p \geq 1$, and validate numerically that the remaining integration region $\left(-\infty,-p \sqrt{\frac{M_{0}\left|\mu^{2}\right|}{4 \lambda a}}\right] \cup\left[p \sqrt{\frac{M_{0}\left|\mu^{2}\right|}{4 \lambda a}},+\infty\right)$ has a negligible contribution to the integration problem. Note that this is the same as to say that the original integration problem over $\mathbb{R}^{d}$ can be truncated satisfactorily for recursive numerical integration to the integration region $\left[-p \sqrt{\frac{M_{0}\left|\mu^{2}\right|}{4 \lambda a}}, p \sqrt{\frac{M_{0}\left|\mu^{2}\right|}{4 \lambda a}}\right]^{d}$. The selection of $p \geq 1$ can be taken to be a positive value that ensures $-\frac{\mu^{2} a^{2}}{M_{0}} x_{i}^{2}-\frac{4 \lambda a^{3}}{M_{0}^{2}} x_{i}^{4} \leq-K$, for some positive value $K$, and $x_{i}$ in $\left(-\infty,-p \sqrt{\frac{M_{0}\left|\mu^{2}\right|}{4 \lambda a}}\right] \cup\left[p \sqrt{\frac{M_{0}\left|\mu^{2}\right|}{4 \lambda a}},+\infty\right)$. By integrating the tails of a normal density $\frac{1}{\sigma \sqrt{2 \pi}} e^{\frac{-t^{2}}{2 \sigma^{2}}}$ outside of the region $|t| \leq 7 \sigma$, we obtain a value below (but close to) $10^{-10}$. The maximal value of the exponential function in the density is given by $e^{-\frac{49}{2}}$ at $t=7 \sigma$. Thus, for our tests we may select the conservative value of $K=24.5$ based on the fact that the tails of the one-dimensional integration problems in the recursive numerical integration method decay faster than a normal density, and that inside of the main importance region the behavior of the marginals seems mainly similar to the one of a shifted normal density with $\sigma=\frac{1}{\sqrt{2}}$ (due to the coupling quadratic term). This choice also makes sense since our computations are carried out in double precision and we aim to obtain at most 10 digits accuracy for the integration problems. Thus, at the end, we select $p \geq 1$ to be the positive number 
that satisfies

$$
\begin{aligned}
-\left(\frac{\mu^{2} a^{2}}{M_{0}}\left(p \sqrt{\frac{M_{0}\left|\mu^{2}\right|}{4 \lambda a}}\right)^{2}+\frac{4 \lambda a^{3}}{M_{0}^{2}}\left(p \sqrt{\frac{M_{0}\left|\mu^{2}\right|}{4 \lambda a}}\right)^{4}\right) & =\left(p^{2}-p^{4}\right)\left(\frac{\left|\mu^{2}\right|^{2} a}{4 \lambda}\right) \\
& =-24.5
\end{aligned}
$$

We choose to take sample sizes $m$ inside of the selected importance region to be small multiples of $\left\lfloor 2 p \sqrt{\left.\frac{M_{0}\left|\mu^{2}\right|}{4 \lambda a}\right\rfloor}\right.$, since we would like to have good integration accuracy in each unit-length interval inside of the parametric problem (10) in the region $\left[-p \sqrt{\frac{M_{0}\left|\mu^{2}\right|}{4 \lambda a}}, p \sqrt{\frac{M_{0}\left|\mu^{2}\right|}{4 \lambda a}}\right]$. Note that in terms of the spacing parameter $a$, we have $m \sim O\left(a^{-\frac{3}{4}}\right)$. The remaining marginal tails outside of $\left[-p \sqrt{\frac{M_{0}\left|\mu^{2}\right|}{4 \lambda a}}, p \sqrt{\frac{M_{0}\left|\mu^{2}\right|}{4 \lambda a}}\right]$ can be estimated with very few Gaussian points (or no points at all) if the relative contribution of the tail of the integrand to the total integral is negligible relative to our maximal target accuracy. For our experiments, we used Gauss-Hermite points (corresponding to a weight function of the form $e^{-c x^{2}}, c>0$ ) and Gaussian points generated from a weight function $e^{-\frac{4 \lambda a^{3}}{M_{0}^{2}} x_{i}^{4}}$, for the integration region $\left(-\infty,-p \sqrt{\frac{M_{0}\left|\mu^{2}\right|}{4 \lambda a}}\right] \cup\left[p \sqrt{\frac{M_{0}\left|\mu^{2}\right|}{4 \lambda a}},+\infty\right)$. By increasing the sample sizes in this region we were able to observe that the relative contribution of the integrand on $\mathbb{R}^{d} \backslash\left[-p \sqrt{\frac{M_{0}\left|\mu^{2}\right|}{4 \lambda a}}, p \sqrt{\frac{M_{0}\left|\mu^{2}\right|}{4 \lambda a}}\right]^{d}$ to the total integrals on $\mathbb{R}^{d}$ seems less than $10^{-12}$. Therefore we believe that the selected importance region for integration can approximate the original problem with great relative accuracy (close to $10^{-11}$ ).

Figur 7 shows our calculations of the two observables $\left\langle x^{2}\right\rangle$ and $\left\langle x^{4}\right\rangle$, depending on the sample size $m$, for different values of lattice points and spacing $(d, a)$. Note that the highest $m \approx 4600$ was taken for $(d, a)=\left(2^{24}, 2^{-12}\right)$, but even in this case the computations took no longer than 3 minutes with a standard PC. For high values of $T$ (like $T=4096$ ) we observe very good results, as well, contrary to what has been observed with randomized QMC in 6. We would like to remark that for the ground state energy $E_{0}$, which, by virtue of the virial theorem, is related to $\left\langle x^{2}\right\rangle$ and $\left\langle x^{4}\right\rangle$ by $E_{0}=\mu^{2} X^{2}+3 \lambda X^{4}+\frac{\mu^{4}}{16}$, the resulting estimates for $T=4096$ matches with the theoretical value in 5 significant digits, $E_{0}=3.8636669$, calculated in [26], namely $\hat{E}_{0}=3.86367053759882$ for $(d, a)=\left(2^{24}, 2^{-12}\right)$ and $m \approx 4600$. 

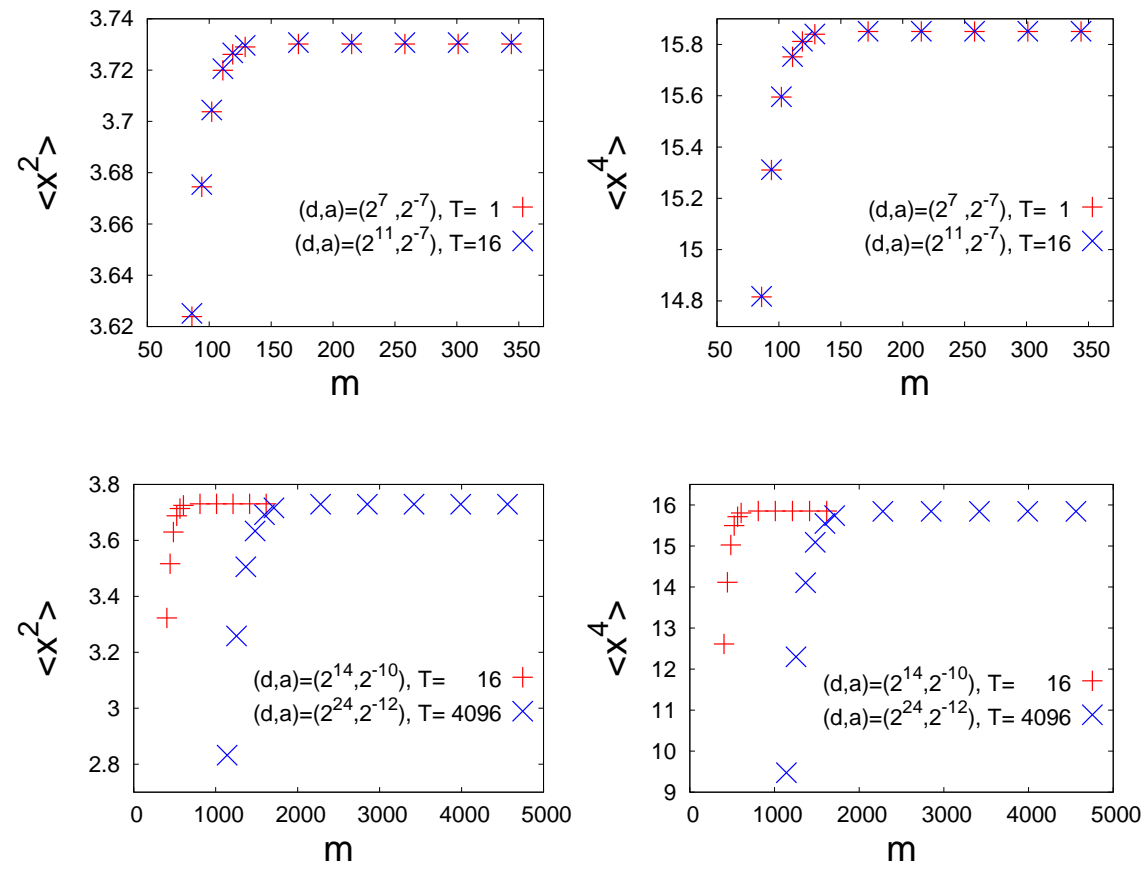

Figure 7: Shown are the estimated values of $\left\langle x^{2}\right\rangle$ (left) and $\left\langle x^{4}\right\rangle$ (right) in the anharmonic oscillator model obtained with recursive Gauss-Legendre quadrature for the pairs $(d, a)=\left(2^{7}, 2^{-7}\right),\left(2^{11}, 2^{-7}\right),\left(2^{14}, 2^{-10}\right)$ and $\left(2^{24}, 2^{-12}\right)$, with corresponding time periods $T=1.0, T=16, T=16$ and $T=4096$. In all cases the relative accuracy achieved with the corresponding maximal samples $m$ seems to be more than 10 significant digits. 


\section{Conclusions}

In this paper we have applied the methods of Quasi Monte Carlo and recursive numerical integration to two quantum mechanical models discretized on a Euclidean time lattice within the path integral approach.

The first model we have considered is a quantum mechanical rotor which is - to some extent - similar to higher dimensional spin systems such as nonlinear $\sigma$-models. For the quantum mechanical rotor we could, unfortunately, not find a successful implementation of the QMC method to solve this model. On the other hand, the method of recursive numerical integration led to a much improved accuracy even when compared to an optimal MCMC algorithm for which we have chosen the cluster algorithm. Conceptually, the error scaling of the recursive numerical integration is at least exponentially fast in the number of integration points $m$. In fig. 5 we could indeed demonstrate that asymptotically this exponential error scaling is realized. Figure 6 shows that even for a small number of integration points the accuracy of the recursive integration method is already much higher than the one of the cluster algorithm with a correspondingly small number of samples.

We remark that we have chosen the cluster algorithm as the MCMC method since it avoids the increase of the autocorrelation time towards the continuum limit, a feature which is shared by the recursive numerical integration technique by construction. The avoidance of autocorrelation times and our finding that a very high accuracy can be reached already for a small number of integration points makes the recursive numerical integration technique a very promising method for more difficult systems where only a small number of samples can be realized, e.g. in higher dimensions.

The other model we looked at is the anharmonic quantum mechanical oscillator. Here, we had found earlier that the QMC method shows an improved error scaling $[6,7,8$. When the extent of the time lattice is kept short, $T \lesssim 1.5$, both, QMC and recursive numerical integration show a comparable performance with an improved error scaling which is faster than $1 / \sqrt{N}$. However, as noted in [7] QMC becomes inefficient when the time extent $T$ is made larger than $T=1.5$, independent of the value of the lattice spacing $a$.

Therefore, we tested the performance of the recursive numerical integration method for various choices of the dimension $d$ and lattice spacing $a$. As fig. 7 shows, we can choose a very broad range for $(d, a)$ where we still find an extremely good performance of the recursive numerical integration method. In fact, the values of the time extent, $T=d a$ can assume very large values such as $T=4096$ and the values of $a$ can become tiny, e.g., $a=2^{-12}$, while still a rapid convergence of the considered quantities is observed.

In conclusion, we have tested two methods to evaluate the path-integral in Euclidean time for quantum mechanical systems. These are the QMC and the recursive numerical integration methods. While we could not find a successful implementation for QMC in the case of the quantum mechanical rotor, the technique of recursive numerical integration has been highly successful with an exponentially fast error scaling. It will be very interesting to test this method for more complicated 1-dimensional models and, of course, for systems in higher dimensions. 


\section{Acknowledgment}

The authors wish to express their gratitude to Prof. Andreas Griewank (Humboldt-Universität zu Berlin) as well as Prof. Michael Müller-Preussker (Humboldt-Universität zu Berlin) for inspiring comments and conversations, which helped to develop the work in this article. H.L., J.V. and K.J. acknowledge financial support by the DFG-funded corroborative research center SFB/TR9 and the DFG projects JA 674/6-1 and GR 705/13.

\section{References}

[1] M. Lüscher, Computational Strategies in Lattice QCD, Lectures given at the Summer School on "Modern perspectives in lattice QCD, Les Houches, August 3-28, 2009 (2010). arXiv:1002.4232

[2] J. Dick, F. Pillichshammer, Digital nets and sequences, Cambridge University Press, Cambridge, 2010, discrepancy theory and quasi-Monte Carlo integration.

[3] J. Dick, F. Y. Kuo, I. H. Sloan, High-dimensional integration: The quasimonte carlo way, Acta Numerica 22 (2013) 133-288. doi:10.1017/ S0962492913000044.

URL http://journals .cambridge.org/article_S0962492913000044

[4] A. Genz, D. K. Kahaner, The numerical evaluation of certain multivariate normal integrals, Journal of Computational and Applied Mathematics 16 (2) (1986) 255-258. doi:10.1016/0377-0427(86)90100-7.

URL http://www.sciencedirect.com/science/article/pii/ 0377042786901007

[5] A. Hayter, Recursive integration methodologies with statistical applications, Journal of Statistical Planning and Inference 136 (7) (2006) 2284-2296, in Memory of Dr. Shanti Swarup Gupta. doi:10.1016/j.jspi.2005.08.024.

URL http://www.sciencedirect.com/science/article/pii/ S0378375805002223

[6] K. Jansen, H. Leovey, A. Nube, A. Griewank, M. Mueller-Preussker, A first look at quasi-Monte Carlo for lattice field theory problems, J.Phys.Conf.Ser. 454 (2013) 012043. arXiv:1211.4388, doi:10.1088/ 1742-6596/454/1/012043.

[7] K. Jansen, H. Leovey, A. Ammon, A. Griewank, M. Muller-Preussker, Quasi-Monte Carlo methods for lattice systems: a first look, Comput.Phys.Commun. 185 (2014) 948-959. arXiv:1302.6419, doi:10.1016/ j.cpc.2013.10.011.

[8] A. Ammon, T. Hartung, K. Jansen, H. Leovey, A. Griewank, et al., Applicability of Quasi-Monte Carlo for lattice systems, PoS LATTICE2013 (2014) 040. arXiv:1311.4726

[9] H. Rothe, Lattice gauge theories: An Introduction, World Sci.Lect.Notes Phys. 43 (1992) 1-381. 
[10] I. Montvay, G. Münster, Quantum fields on a lattice, Cambridge Monographs on Mathematical Physics, Cambridge University Press, 1994.

[11] C. Gattringer, C. B. Lang, Quantum chromodynamics on the lattice, Lect.Notes Phys. 788 (2010) 1-211. doi:10.1007/978-3-642-01850-3.

[12] I. M. Sobol', The distribution of points in a cube and the approximate evaluation of integrals, U.S.S.R. Comput. Math. and Math. Phys. 7 (4) (1967) 86-112.

[13] A. B. Owen, Randomly Permuted $(t, m, s)$-Nets and $(t, s)$-Sequences, in: H. Niederreiter, P. J.-S. Shiue (Eds.), Monte Carlo and Quasi-Monte Carlo Methods in Scientific Computing, Vol. 106 of Lecture Notes in Statistics, Springer-Verlag, 1995, pp. 299-317.

[14] L. Devroye, Non-Uniform Random Variate Generation, Springer-Verlag, 1986.

[15] D. B. Rubin, The calculation of posterior distributions by data augmentation. Comment: a noniterative sampling/importance resampling alternative to the data augmentation algorithm for creating a few imputations when fractions of missing information are modest: the SIR algorithm, Journal of the American Statistical Association 82 (398) (1987) 543-546.

[16] D. B. Rubin, Using the sir algorithm to simulate posterior distributions, in: J. Bernardo, M. DeGroot, D. Lindley, A. Smith (Eds.), Bayesian Statistics, Vol. 3, Oxford University Press, Oxford, 1988, pp. 395-402.

[17] N. J. Gordon, D. J. Salmond, A. F. M. Smith, Novel approach to nonlinear/non-Gaussian Bayesian state estimation, IEE Proceedings-F 140 (2) (1993) 107-113.

[18] B. Vandewoestyne, R. Cools, On the convergence of quasi-random sampling/importance resampling, Mathematics and Computers in Simulation $81(2010)$ 490-505.

[19] J. von Neumann, Various techniques used in connection with random digits. Monte Carlo methods, Nat. Bureau Standards 12 (1951) 36-38.

[20] B. Moskowitz, R. E. Caflisch, Smoothness and Dimension Reduction in Quasi-Monte Carlo Methods, Mathl. Comput. Modelling 23(8/9) (1996) $37-54$.

[21] Y. Saad, Numerical Methods for Large Eigenvalue Problems, Society for Industrial and Applied Mathematics, 2011. arXiv:http://epubs.siam.org/ doi/pdf/10.1137/1.9781611970739, doi:10.1137/1.9781611970739 URL http://epubs . siam.org/doi/abs/10.1137/1.9781611970739

[22] T. DeGrand, C. E. Detar, Lattice methods for quantum chromodynamics, World Scientific Publishing, 2006.

[23] F. Niedermayer, Cluster algorithms, Lect.Notes Phys. 501 (1998) 36. arXiv:hep-lat/9704009, doi:10.1007/BFb0105458. 
[24] N. Metropolis, A. Rosenbluth, M. Rosenbluth, A. Teller, E. Teller, Equation of state calculations by fast computing machines, J. Chem. Phys. 21 (1953) 1087-1092.

[25] W. Bietenholz, U. Gerber, M. Pepe, U.-J. Wiese, Topological Lattice Actions, JHEP 1012 (2010) 020. arXiv:1009.2146, doi:10.1007/ JHEP12(2010) 020

[26] R. Blankenbecler, T. A. DeGrand, R. Sugar, Moment Method for Eigenvalues and Expectation Values, Phys.Rev. D21 (1980) 1055. doi:10.1103/ PhysRevD.21.1055. 